\title{
Optimizing the diagnosis and management of dementia within primary care: a systematic review of systematic reviews
}

Brooklynn Fernandes ${ }^{1 *}$, Zahra Goodarzi ${ }^{2}$ and Jayna Holroyd-Leduc ${ }^{2}$

\begin{abstract}
Background: To understand how best to approach dementia care within primary care and its challenges, we examined the evidence related to diagnosing and managing dementia within primary care.

Methods: Databases searched include: MEDLINE, Embase, PsycINFO and The Cochrane Database of Systematic Reviews from inception to 11 May 2020. English-language systematic reviews, either quantitative or qualitative, were included if they described interventions involving the diagnosis, treatment and/or management of dementia within primary care/family medicine and outcome data was available. The risk of bias was assessed using AMSTAR 2 . The review followed PRISMA guidelines and is registered with Open Science Framework.
\end{abstract}

Results: Twenty-one articles are included. The Mini-Cog and the MMSE were the most widely studied cognitive screening tools. The Abbreviated Mental Test Score (AMTS) achieved high sensitivity (100\%, $95 \%$ Cl: 70-100\%) and specificity ( $82 \%, 95 \% \mathrm{Cl}: 72-90 \%)$ within the shortest amount of time (3.16 to $5 \mathrm{~min}$ ) within primary care. Five of six studies found that family physicians had an increased likelihood of suspecting dementia after attending an educational seminar. Case management improved behavioural symptoms, while decreasing hospitalization and emergency visits. The primary care educational intervention, Enhancing Alzheimer's Caregiver Health (Department of Veterans Affairs), was successful at increasing carer ability to manage problem behaviours and improving outcomes for caregivers.

Conclusions: There are clear tools to help identify cognitive impairment in primary care, but strategies for management require further research. The findings from this systematic review will inform family physicians on how to improve dementia diagnosis and management within their primary care practice.

Keywords: Dementia, Primary care, Family physician, Systematic review, Diagnosis

*Correspondence: Brooklynn.fernand1@ucalgary.ca

1 Faculty of Science, University of Calgary, Calgary, Canada

Full list of author information is available at the end of the article

(c) The Author(s). 2021. Open Access This article is licensed under a Creative Commons Attribution 4.0 International License, which permits use, sharing, adaptation, distribution and reproduction in any medium or format, as long as you give appropriate credit to the original author(s) and the source, provide a link to the Creative Commons licence, and indicate if changes were made. The images or other third party material in this article are included in the article's Creative Commons licence, unless indicated otherwise in a credit line to the material. If material is not included in the article's Creative Commons licence and your intended use is not permitted by statutory regulation or exceeds the permitted use, you will need to obtain permission directly from the copyright holder. To view a copy of this licence, visit http://creativecommons.org/licenses/by/4.0/. The Creative Commons Public Domain Dedication waiver (http://creativeco $\mathrm{mmons}$.org/publicdomain/zero/1.0/) applies to the data made available in this article, unless otherwise stated in a credit line to the data. 


\section{Background}

At any given time, 5-8\% of the general population aged 60 and over are living with dementia, and it is expected that 152 million people in the world will have dementia by 2050 [1]. The impact of dementia is far reaching, as it affects not only the person with dementia, but also their family carers, the healthcare system and society as a whole [1]. Dementia is often unrecognized, and there is an underuse of diagnostic assessment tools and a lack of attention to the issues faced by family caregivers [2]. Approximately $65 \%$ of dementia cases are undiagnosed in primary care, which negatively impacts these patients by not implementing advanced care planning and management strategies before the dementia progresses [3]. The U.S Preventative Services Task Force recommends that clinicians assess cognitive functioning when a patient is suspected of cognitive impairment based on the physician's observation or caregiver concerns [3]. Canadian consensus guidelines similarly do not recommend asymptomatic screening, but instead suggest use of validated screening tools if there is clinical concern for a cognitive disorder [4]. Common neuropsychological screening tools administered by family physicians (FPs) include the Mini-Mental State Examination (MMSE) and Clock Drawing Test (CDT) [3]. However, it is not clear that these are the best screening tools for use in primary care.

Time constraints are often an issue for family doctors as it relates to diagnosing and managing dementia. The time allocated for a typical office visit makes it challenging to perform a cognitive assessment [5]. FPs often feel uncertainty regarding the management of dementia after a diagnosis has been made [5]. This highlights the current need to better optimize dementia care within primary care. The objective of this systematic review of systematic reviews was to determine the most effective evidence-based strategies to diagnose and manage dementia within primary care. Specifically, we seek to understand what practices FPs can undertake to ensure accurate and timely testing and management.

\section{Methods}

This systematic review was conducted in accordance to PRISMA (Preferred Reporting Items for Systematic Reviews and Meta-analyses) guidelines [6], and the protocol is registered in Open Science Framework [DOI https://doi.org/10.17605/OSF.IO/E4AW5]. All data generated or analysed during this study are included in this published article in Additional file 1: Appendixes 1 and 2. A systematic review of systematic reviews was determined to be the past method to further summarize and tailor the current body of literature on this topic into a format that would address the existing evidence to practice gap.

\section{Data Sources}

The systematic literature search was developed in consultation with a health sciences librarian, with the final search being completed 11 May 2020. The following databases using the Ovid platform were searched without a restriction to publication date: MEDLINE, EMBASE, PsycINFO and The Cochrane Database of Systematic Reviews. We searched the following clusters of search terms: Family Practice and Dementia. In each category, we used controlled vocabulary such as Medical Subject Headings (MeSH) as well as keywords. Within each cluster, terms were combined with OR, and between the clusters with AND. We then used CADTH search terms for the systematic review study designs [7] (Additional file 1: Appendix 1). The reference list of a previous relevant systematic review of systematic reviews published in 2014 was also searched [8].

\section{Study Selection}

Systematic reviews were considered if they met the following inclusion criteria.

- Population: Primary care or family practice settings seeing persons with dementia.

- Intervention: The detection, diagnosis, treatment and/or management of dementia including models of care, pathways and/or protocols.

- Comparators: Usual care, wait-list control or other interventions within the scope of the review.

- Outcomes: The description of the detection, diagnosis, treatment or management strategies, along with measures of their acceptability, efficacy or effectiveness in the provision of care.

- Study design: Systematic review, either quantitative or qualitative.

Articles were also selected for inclusion if they were English-language articles, included relevant descriptions of the interventions used, and outcome data was available.

Two reviewers (B.F and J.H.-L.) independently screened the titles and abstracts for possible inclusion. If either reviewer thought the citation was relevant or potentially relevant, the full-text article was then retrieved for further evaluation. All full-text articles were assessed independently for inclusion by B.F and J.H.-L. Any conflicts were resolved through discussion. One reviewer (B.F.) independently extracted the following information from the included full-text studies using a standardized data extraction form: authors, year of publication, country where the review was conducted, number of studies 
included, study designs included, databases searched, time frame of article search, inclusion and exclusion criteria, population (mean age, SD and dementia diagnosis), intervention, comparator, sample size, setting (if the intervention was cognitive screening, the method of administration), time of administration (if intervention was cognitive screening), cognitive outcome(s) measured, results (meta-analysis, Sn, Sp, accuracy), and other (Additional file 1: Appendix 2). One reviewer (B.F) categorized each study based on the primary category of intervention, which was verified by another reviewer (J.H-L).

\section{Quality Assessment and Analysis}

Two reviewers (B.F and J.H.-L.) independently assessed the quality of the included studies using the AMSTAR 2 Systematic Review Quality Appraisal Checklist 2020. Systematic reviews without a clear PICO were excluded. Best practices for quality assessment using AMSTAR 2 are to consider the impact of inadequate ratings for each category rather than generate an overall score. The AMSTAR 2 quality appraisal results for each of the included studies is available in Additional file 1: Appendix 3 [9]. A qualitative descriptive summary of the literature is presented.

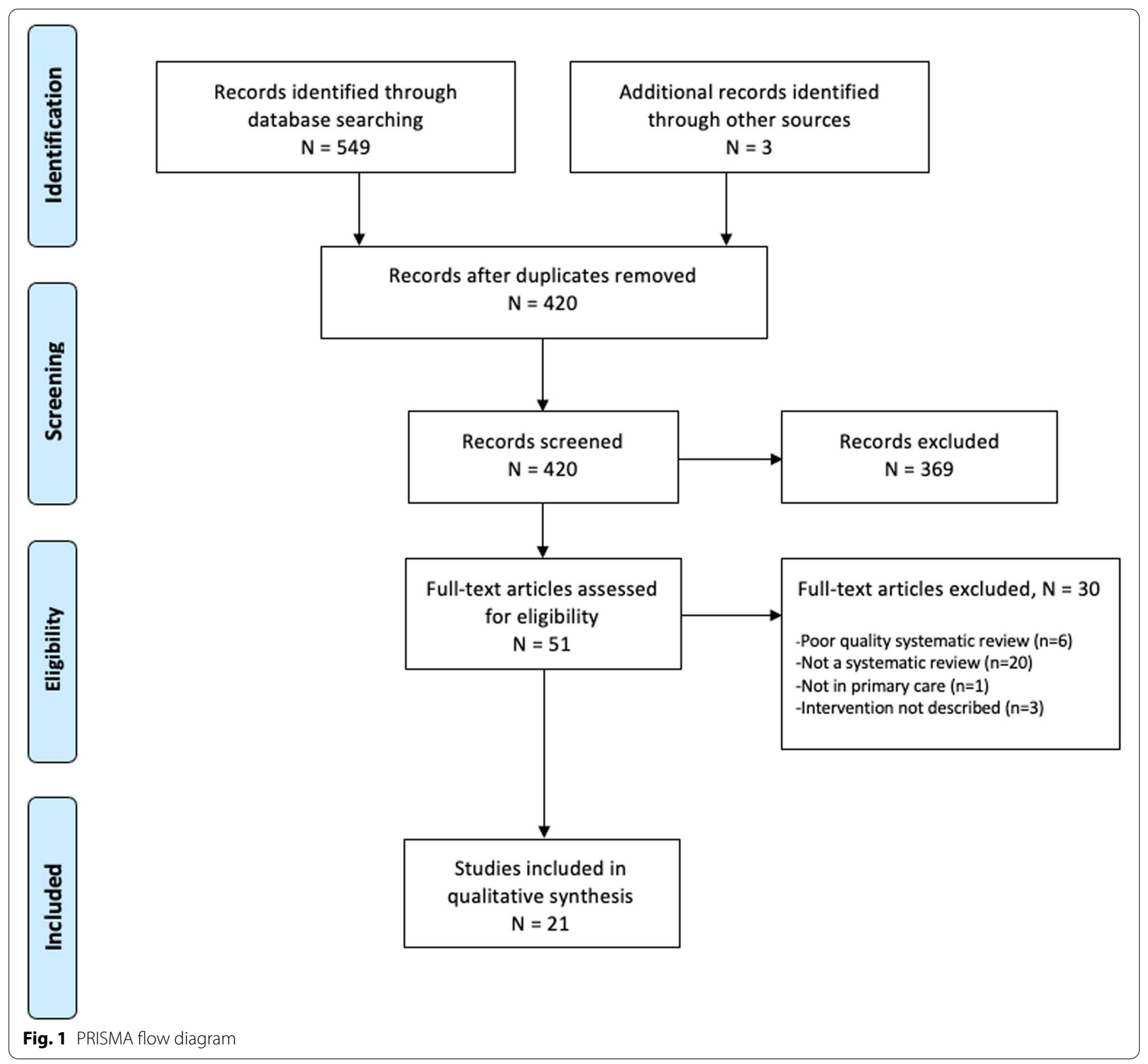


Table 1 Screening tools and their comparators, cognitive outcomes, administration time, sensitivity and specific and conclusions from the literature included in this systematic review

\begin{tabular}{|c|c|c|c|c|c|c|c|c|c|}
\hline $\begin{array}{l}\text { Reference, } \\
\text { Country }\end{array}$ & $\begin{array}{l}\text { Number } \\
\text { of studies } \\
\text { included in } \\
\text { systematic } \\
\text { review }\end{array}$ & Intervention(s) & Comparator & $\begin{array}{l}\text { Cognitive } \\
\text { outcome(s) } \\
\text { measured }\end{array}$ & $\begin{array}{l}\text { Time of } \\
\text { administration } \\
\text { (minutes) }\end{array}$ & Sensitivity (\%) & Specificity & Conclusions & Abbreviations \\
\hline $\begin{array}{l}\text { Mitchell et al, } \\
\text { United } \\
\text { Kingdom }\end{array}$ & 44 & $\begin{array}{l}\text { Multidomain } \\
\text { screening tests } \\
\text { (known as a } \\
\text { battery detec- } \\
\text { tion method) } \\
\text { in primary care } \\
\text { which assess for } \\
\text { multiple cogni- } \\
\text { tive domains. } \\
\text { Primary care } \\
\text { case-finding }+ \text { : } \\
\text {-AMTS/MSQ } \\
\text {-MSQ } \\
\text {-WIND-SET } \\
\text {-PCL } \\
\text {-AMTS } \\
\text {-PCL } \\
\text { Primary care } \\
\text { Screening } \neq: \\
\text { - PCL } \\
\text {-AMTS/MSQ } \\
\text {-MSQ } \\
\text {-SPMSQ } \\
\text {-GPCOG }\end{array}$ & MMSE & Dementia & $\begin{array}{l}\text { Primary care case- } \\
\quad \text { finding: } \\
\text {-AMTS/MSQ }=4 \\
\text { - } \mathrm{MSQ}=2 \\
\text {-WIND-SET }=1 \\
\text {-PCL }=11 \\
\text { - } \mathrm{AMTS}=2 \\
\text {-PCL }=11 \\
\text { Primary care } \\
\quad \text { screening: } \\
\text { - } \mathrm{PCL}=11 \\
\text {-AMTS/MSQ }=4 \\
\text {-MSQ }=2 \\
\text {-SPMSQ }=2 \\
\text {-GPCOG }=5 \\
\text { Comparator: } \\
\text {-MMSE }=9 \\
\quad \text { with healthy } \\
\text { individuals and } \\
15 \text { with patients } \\
\text { with dementia. }\end{array}$ & $\begin{array}{l}\text { Battery } \\
\text { detection } \\
\text { methods: } \\
\text {-84.0 (95\% Cl } \\
74.2-91.8)\end{array}$ & $\begin{array}{l}\text { Battery detec- } \\
\text { tion methods: } \\
\text {-89.9 (95\% Cl } \\
78.3-97.4)\end{array}$ & $\begin{array}{l}\text { The optimal } \\
\text { individual tools } \\
\text { were the AMTS/ } \\
\text { MSQ and PCL. } \\
\text { AMTS was } \\
\text { superior to the } \\
\text { MMSE for case } \\
\text { finding however } \\
\text { the MMSE was } \\
\text { optimal for } \\
\text { screening. }\end{array}$ & $\begin{array}{l}\text { AMTS/MSQ-Abbreviated } \\
\text { Mental Test Score/Men- } \\
\text { tal Status Questionnaire, } \\
\text { (WIND-SET)-Specific Set } \\
\text { of items from MMSE, } \\
\text { PCL-Prueba cognitive } \\
\text { de leganes, AMTS- } \\
\text { Abbreviated mental test } \\
\text { score, GPCOG-General } \\
\text { practitioner's assess- } \\
\text { ment of cognition, } \\
\text { MMSE-Mini-Mental } \\
\text { State Examination } \\
\text { † Case-finding is defined } \\
\text { as any tool or question- } \\
\text { naire which identifies a } \\
\text { condition with minimal } \\
\text { false negatives, meas- } \\
\text { ured as the positive } \\
\text { predicative value. } \\
\text { ₹ Screening is the ability } \\
\text { of a test to rule out a } \\
\text { diagnosis with minimal } \\
\text { false positives, reported } \\
\text { as the negative predic- } \\
\text { tive value. }\end{array}$ \\
\hline $\begin{array}{l}\text { Creavin et al, } \\
\text { United } \\
\text { Kingdom }\end{array}$ & 70 & -MMSE & $\begin{array}{l}\text { A commonly } \\
\text { accepted } \\
\text { clinical (gold) } \\
\text { reference } \\
\text { standard. }\end{array}$ & Dementia & $\begin{array}{l}\text {-MMSE=7 with } \\
\text { a patient with } \\
\text { dementia and } \\
5 \text { with a person } \\
\text { with normal } \\
\text { cognition }\end{array}$ & $\begin{array}{l}\text { Carnero-Pardo } \\
\text { 2013: } \\
\text {-Cut point } \\
\text { of } 17= \\
70(95 \% \mathrm{Cl} \\
59-80) \\
\text { - Cut point of } \\
24=100 \\
(95 \% \mathrm{Cl} \\
95-100)\end{array}$ & $\begin{array}{l}\text { Carnero-Pardo } \\
\text { 2013: } \\
\text {-Cut point of } 17 \\
=93(95 \% \mathrm{Cl} \\
89,96) \\
\text {-Cut point of } 24 \\
=46(95 \% \mathrm{Cl} \\
40-52)\end{array}$ & $\begin{array}{l}\text { Carnero-Pardo } 2013 \\
\text { reported there } \\
\text { were some false } \\
\text { negatives as the } \\
\text { sensitivity fell } \\
\text { from } 1.00(95 \% \\
\text { Cl } 0.95 \text { to } 1.00) \\
\text { to } 0.70(95 \% \mathrm{Cl} \\
0.59 \text { to } 0.80) \text {. The } \\
\text { summary diag- } \\
\text { nostic accuracy } \\
\text { could not be } \\
\text { estimated due to } \\
\text { insufficient data. }\end{array}$ & \\
\hline
\end{tabular}


Table 1 (continued)

\begin{tabular}{|c|c|c|c|c|c|c|c|c|c|}
\hline $\begin{array}{l}\text { Reference, } \\
\text { Country }\end{array}$ & $\begin{array}{l}\text { Number } \\
\text { of studies } \\
\text { included in } \\
\text { systematic } \\
\text { review }\end{array}$ & Intervention(s) & Comparator & $\begin{array}{l}\text { Cognitive } \\
\text { outcome(s) } \\
\text { measured }\end{array}$ & $\begin{array}{l}\text { Time of } \\
\text { administration } \\
\text { (minutes) }\end{array}$ & Sensitivity (\%) & Specificity & Conclusions & Abbreviations \\
\hline $\begin{array}{l}\text { Abd Razak } \\
\text { et al, } \\
\text { Malaysia }\end{array}$ & 30 & $\begin{array}{l}\text {-MoCA-B; MoCA } \\
\text {-SPMSQ } \\
\text {-MEFO } \\
\text {-ACE-III } \\
\text {-AQT-CF } \\
\text {-SLUMS } \\
\text {-5 Object Test } \\
\text {-BNB Semantic } \\
\quad \text { Fluency } \\
\text {-SMCC compared } \\
\quad \text { to MMSE and } \\
\text { CDT } \\
\text {-CASI-S } \\
\text {-RCS } \\
\text {-CPS } \\
\text {-Literacy Inde- } \\
\quad \text { Pendent Cogni- } \\
\text { tive Assessment } \\
\text {-BIMS; BCAT } \\
\text {-3MS } \\
\text {-Mini-Cog; MIS; } \\
\quad \text { MF-2 } \\
\text {-VT-VSM;VR-DOT } \\
\text {-CCS } \\
\text {-CAMCI } \\
\text {-CADi;CADi-2 } \\
\text {-DRA } \\
\text {-p-AD8 } \\
\text { - IQCODE }\end{array}$ & $\begin{array}{l}\text { Comparing the } \\
\text { feasibility } \\
\text { and validity } \\
\text { between } \\
\text { the various } \\
\text { screening } \\
\text { tools. }\end{array}$ & $\begin{array}{l}\text { Mild cognitive } \\
\text { impair- } \\
\text { ment and } \\
\text { dementia }\end{array}$ & 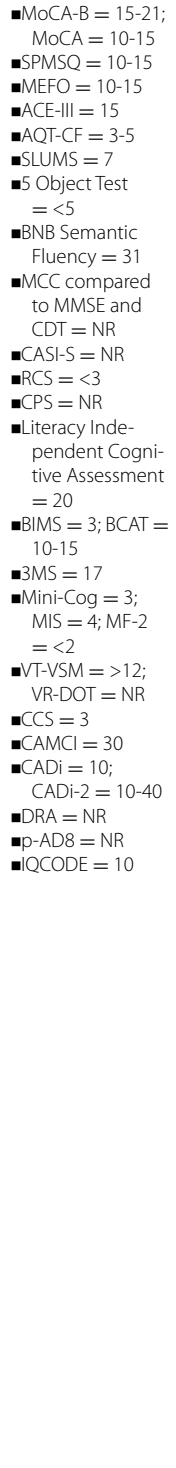 & $\begin{array}{l}\text { For detecting } \\
\text { dementia: } \\
\text {-ACE-III at a } \\
\text { cut-off point } \\
\text { of }<81 \text {, Sn } \\
=100 \\
\text { For detecting } \\
\text { MCl: } \\
\text {-MoCA, Sn }= \\
\text { 91-97 }\end{array}$ & $\begin{array}{l}\text { For detecting } \\
\text { dementia: } \\
\text {-ACE-III at a cut- } \\
\text { off point of } \\
<81,5 p=96 \\
\text { For detecting } \\
\text { MCl: } \\
\text {-MoCA, Sp = } \\
60-80\end{array}$ & $\begin{array}{l}\text { For detecting } \\
\text { dementia: } \\
\text { Screening tools } \\
\text { less sensitive to } \\
\text { ACE-IIl but with } \\
\text { relatively high } \\
\text { Sn/Sp values } \\
\text { were: } \\
\text { SLUMS, RCS, and } \\
\text { BCAT. } \\
\text { For detecting } \\
\text { MCl: The MoCA } \\
\text { was the most } \\
\text { commonly used } \\
\text { tool and had the } \\
\text { highest Sn/Sp } \\
\text { ranges. } \\
\text { Less specific to } \\
\text { the MoCA but } \\
\text { among the } \\
\text { most sensitive } \\
\text { tools were the } \\
\text { (VR-DOT) and } \\
\text { IQCODE. Tools } \\
\text { with the highest } \\
\text { specificity but } \\
\text { with lower } \\
\text { sensitivity were: } \\
\text { The } 5 \text { Objects } \\
\text { Test, RCS, CPS, } \\
\text { and (VT-VSM). }\end{array}$ & $\begin{array}{l}\text { NR-Not Reported, } \\
\text { MCI-Mild Cognitive } \\
\text { Impairment, (MoCA- } \\
\text { B)-Montreal Cognitive } \\
\text { Assessment-Basic, } \\
\text { (MoCA)-Montreal } \\
\text { Cognitive Assessment, } \\
\text { SPMSQ-Short Portable } \\
\text { Mental Status Question- } \\
\text { naire, (MEFO)-Memory, } \\
\text { fluency and orientation, } \\
\text { (ACE-III)-Addenbrooke's } \\
\text { Cognitive Examination } \\
\text { III, (AQT-CF)-A Quick } \\
\text { Test of Cognitive Speed, } \\
\text { (SLUMS)- Saint Louis } \\
\text { University Mental } \\
\text { Status, (BNB)-Brief } \\
\text { Neuropsychological Bat- } \\
\text { tery Semantic Fluency, } \\
\text { (SMCC)-The Subjective } \\
\text { Memory Complaint } \\
\text { Clinical, (CASI-S)-Cogni- } \\
\text { tive Abilities Screening } \\
\text { Instrument-Short, } \\
\text { (RCS)-Rapid Cognitive } \\
\text { Screen, (CPS)-Cognitive } \\
\text { Performance Scale, } \\
\text { (BIMS)-Brief Interview } \\
\text { for Mental Status, } \\
\text { (BCAT)-Brief Cognitive } \\
\text { Assessment Tool, (3MS)- } \\
\text { Modified Mini-Mental } \\
\text { State Examination, } \\
\text { (MIS)-Memory } \\
\text { Impairment Screen, } \\
\text { (MF-2)-Memory Func- } \\
\text { tion 2, (VT-VSM)-Virtual } \\
\text { Reality technology: } \\
\text { Virtual supermarket, } \\
\text { (VR-DOT)-Virtual Reality } \\
\text { Day-Out-Task, (CCS)- } \\
\text { Computerized Cognitive } \\
\text { Screening Tests, } \\
\text { (CAMCI)-Computerized } \\
\text { Assessment of Mild } \\
\text { Cognitive Impairment, } \\
\text { (CADi)-[Cognitive } \\
\text { Assessment for } \\
\text { Dementia, iPad version], } \\
\text { (CADi-2)-[Revised } \\
\text { Cognitive Assessment } \\
\text { for Dementia, iPad ver- } \\
\text { sion], (DRA)-Dementia } \\
\text { Risk Assessment, } \\
\text { (p-AD8)-Participant- } \\
\text { rated, (IQCODE)-Inform- } \\
\text { ant Questionnaire on } \\
\text { Cognitive Decline in the } \\
\text { Elderly individuals }\end{array}$ \\
\hline $\begin{array}{l}\text { Smith et al, } \\
\text { United } \\
\text { Kingdom }\end{array}$ & 33 & $\begin{array}{l}\text {-Rural Older Adult } \\
\text { Memory Evalu- } \\
\text { ation } \\
\text {-Mini-Cog } \\
\text {-PRISM-PC } \\
\text {-SAPH question- } \\
\text { naire } \\
\text {-MMSE and clinical } \\
\text { history/exami- } \\
\text { nation } \\
\text {-7-minute screen } \\
\text {-CIE and MMSE }\end{array}$ & Not mentioned. & Dementia & Not mentioned. & $\begin{array}{l}\text { Not men- } \\
\text { tioned. }\end{array}$ & Not mentioned. & $\begin{array}{l}\text { There is insufficient } \\
\text { evidence to sup- } \\
\text { port the adop- } \\
\text { tion of these } \\
\text { programmes into } \\
\text { practice. Six posi- } \\
\text { tive and eight } \\
\text { negative effects } \\
\text { of primary care } \\
\text { screening and } \\
\text { early diagnosis of } \\
\text { dementia were } \\
\text { reported. }\end{array}$ & $\begin{array}{l}\text { (PRISM-PC)-Perceptions } \\
\text { Regarding Investiga- } \\
\text { tional Screening for } \\
\text { Memory in Primary } \\
\text { Care, SAPH-Dementia } \\
\text { Screening and Per- } \\
\text { ceived Hames, CIE-The } \\
\text { Canberra Interview for } \\
\text { the Elderly }\end{array}$ \\
\hline
\end{tabular}


Table 1 (continued)

\begin{tabular}{|c|c|c|c|c|c|c|c|c|c|}
\hline $\begin{array}{l}\text { Reference, } \\
\text { Country }\end{array}$ & $\begin{array}{l}\text { Number } \\
\text { of studies } \\
\text { included in } \\
\text { systematic } \\
\text { review }\end{array}$ & Intervention(s) & Comparator & $\begin{array}{l}\text { Cognitive } \\
\text { outcome(s) } \\
\text { measured }\end{array}$ & $\begin{array}{l}\text { Time of } \\
\text { administration } \\
\text { (minutes) }\end{array}$ & Sensitivity (\%) & Specificity & Conclusions & Abbreviations \\
\hline $\begin{array}{c}\text { Brodaty et al, } \\
\text { Australia }\end{array}$ & 83 & $\begin{array}{l}\text { Instruments } \\
\text { Validated in } \\
\text { General Practice, } \\
\text { Community } \\
\text { or Population } \\
\text { Samples: } \\
\text {-AMT } \\
\text {-Cambridge } \\
\text { Cognitive } \\
\text { Examination } \\
\text {-CDT } \\
\text {-GPCOG } \\
\text {-Mini-Cog } \\
\text {-MIS } \\
\text {-MMSE } \\
\text {-Short and Sweet } \\
\text { Screening } \\
\text { Instrument } \\
\text {-Short IQCODE }\end{array}$ & MMSE & Dementia & $\begin{array}{l}\text {-AMT }=3: 16 \\
\text {-Camnridge Cog- } \\
\quad \text { nitive Examina- } \\
\text { tion }=20 \\
\text {-CDT }=2: 16 \\
\text {-GPCOG }=4.5 \\
\text {-Mini-Cog }=2-4 \\
\text {-MIS }=4 \\
\text {-MMSE }=4 \\
\text {-Short and Sweet } \\
\quad \text { Screening } \\
\text { Instrument } \\
=10 \\
\text {-Short IQCODE } \\
=30 \mathrm{~s}\end{array}$ & $\begin{array}{l}\text { Screening tests } \\
\text { validated } \\
\text { in general } \\
\text { practice, } \\
\text { community } \\
\text { or popula- } \\
\text { tion samples: } \\
\text {-AMT-100 (95\% } \\
\text { Cl 70-100) } \\
\text {-Cambridge } \\
\text { Cognitive } \\
\text { Examina- } \\
\text { tion-88 (95\% } \\
\text { CI 64-99) } \\
\text {-CDT-76 (95\% } \\
\text { CI 60-88) } \\
\text {-GPCOG-85 } \\
\text { (95\% Cl } \\
76-92) \\
\text {-Mini-Cog-76 } \\
\text { (95\% Cl } \\
65-85) \\
\text {-MIS-80 (95\% } \\
\text { Cl 66-90) } \\
\text {-MMSE-69 } \\
\text { (95\% Cl } \\
66-73) \\
\text {-Short and } \\
\text { Sweet } \\
\text { Screening } \\
\text { Instru- } \\
\text { ment-94 } \\
\text { (95\% Cl } \\
88-96) \\
\text {-Short } \\
\text { IQCODE-79 } \\
\text { (95\% Cl } \\
65-90)\end{array}$ & $\begin{array}{l}\text { Screening tests } \\
\text { validated } \\
\text { in general } \\
\text { practice, } \\
\text { community } \\
\text { or population } \\
\text { samples: } \\
\text {-AMT-82 (95\% } \\
\text { Cl 72-90) } \\
\text {-Cambridge } \\
\text { Cognitive } \\
\text { Examina- } \\
\text { tion-75 (95\% } \\
\text { Cl 67-83) } \\
\text {-CDT-81 (95\% CI } \\
\text { 77-84) } \\
\text {-GPCOG-86 } \\
\text { (95\% CI } \\
\text { 81-91) } \\
\text {-Mini-Cog-89 } \\
\text { (95\% Cl } \\
87-91) \\
\text {-MIS-96 (95\% CI } \\
\text { 94-98) } \\
\text {-MMSE-89 (95\% } \\
\text { Cl 87-92) } \\
\text {-Short and } \\
\text { Sweet Screen- } \\
\text { ing Instru- } \\
\text { ment-91 (95\% } \\
\text { CI 90-92) } \\
\text {-Short } \\
\text { IQCODE-82 } \\
\text { (95\% CI } \\
79-85 \text { ) }\end{array}$ & $\begin{array}{l}\text { Screening tests } \\
\text { validated in } \\
\text { general practice, } \\
\text { community } \\
\text { or population } \\
\text { samples: } \\
\text { AMT had a } \\
\text { PPV }=0.42 \text { (95\% } \\
\text { CI), NPV=1.00 } \\
\text { (95\% CI), misclas- } \\
\text { sification of 16\%, } \\
\text { had internal } \\
\text { consistency and } \\
\text { face validity. } \\
\text { Mini-Cog had a } \\
\text { PPV=0.34 (95\% } \\
\text { CI), NPV=0.98 } \\
\text { (95\% CI), } 12 \% \\
\text { misclassification, } \\
\text { no education } \\
\text { bias or language/ } \\
\text { cultural bias, } \\
\text { and had face } \\
\text { validity*. } \\
\text { The AMT, CDT, } \\
\text { GPCOG, Short } \\
\text { IQCODE, Mini- } \\
\text { Cog, and MIS all } \\
\text { had a NPV =< } \\
\text { MMSE (0.92). } \\
\text { The GPCOG, } \\
\text { Mini-Cog and } \\
\text { MIS had a } \\
\text { misclassification } \\
\text { rate }=<\text { MMSE } \\
\text { (15\%) and had a } \\
\text { high sensitivity } \\
\text { and specificity } \\
\text { (>=80\%) and } \\
\text { were therefore } \\
\text { chosen as the } \\
\text { most suitable } \\
\text { instruments for } \\
\text { use in general } \\
\text { practice. }\end{array}$ & $\begin{array}{l}\text { MAT-Mental Alterna- } \\
\text { tion Test. *- (Based } \\
\text { on Diagnostic and } \\
\text { Statistical Manual } \\
\text { of Mental Disorders, } \\
\text { Fourth Edition criteria } \\
\text { requiring that instru- } \\
\text { ments test memory } \\
\text { and at least one other } \\
\text { cognitive domain). } \\
\text { CDT-Clock Drawing } \\
\text { Test. GPCOG-General } \\
\text { Practitioner Assessment } \\
\text { of Cognition. }\end{array}$ \\
\hline $\begin{array}{r}\text { Seitz et al, } \\
\text { Canada }\end{array}$ & 4 & $\begin{array}{l}\text { The Mini-Cog } \\
\text { performed in } \\
\text { insolation or } \\
\text { scored based on } \\
\text { results on the } \\
\text { CDT or three- } \\
\text { word recall }\end{array}$ & $\begin{array}{l}\text { Standard diag- } \\
\text { nostic criteria } \\
\text { for the clinical } \\
\text { diagnosis of } \\
\text { dementia }\end{array}$ & $\begin{array}{l}\text { Alzheimer's } \\
\text { disease } \\
\text { dementia } \\
\text { and related } \\
\text { dementias }\end{array}$ & $\begin{array}{l}\text { Mini-Cog }=3-5 \text { in } \\
\text { routine practice }\end{array}$ & $\begin{array}{l}\text { Carnero- } \\
\text { Pardo } 2013 \\
\text { dementia } \\
\text { prevalence } \\
\text { was 34.5\%: } \\
\text {-100 (95\% Cl } \\
93-100) \\
\text { Fuchs 2012 } \\
5.0 \% \\
\text { dementia } \\
\text { prevalence: } \\
\text {-100 (95\% Cl } \\
84-100) \\
\text { Holsinger 2012 } \\
\text { (highest } \\
\text { quality } \\
\text { study) } 5.5 \% \\
\text { dementia } \\
\text { prevalence: } \\
\text {-76 (95\% Cl } \\
53-92) \\
\text { McCarten } \\
201290.3 \% \\
\text { dementia } \\
\text { prevalence: } \\
\text {-84 (95\% Cl } \\
81-87 \text { ) }\end{array}$ & $\begin{array}{l}\text { Carnero-Pardo } \\
\text { 2013: } \\
\text {-40 (95\% Cl } \\
\quad 30-50) \\
\text { Fuchs 2012: } \\
\text {-85 (95\% Cl } \\
\text { 81-89) } \\
\text { Holsinger 2012: } \\
\text { - } 73 \text { (95\% Cl } \\
\quad 68-77) \\
\text { McCarten 2012: } \\
\text { - 27 (95\% Cl } \\
\text { 16-41) }\end{array}$ & $\begin{array}{l}\text { Presently there } \\
\text { is insufficient } \\
\text { evidence to } \\
\text { support the use } \\
\text { of Mini-Cog in } \\
\text { primary care. } \\
\text { Studies mentioned } \\
\text { are primary } \\
\text { journal articles } \\
\text { (cross-sectional } \\
\text { studies). }\end{array}$ & \\
\hline
\end{tabular}


Table 1 (continued)

\begin{tabular}{|c|c|c|c|c|c|c|c|c|c|}
\hline $\begin{array}{l}\text { Reference, } \\
\text { Country }\end{array}$ & $\begin{array}{l}\text { Number } \\
\text { of studies } \\
\text { included in } \\
\text { systematic } \\
\text { review }\end{array}$ & Intervention(s) & Comparator & $\begin{array}{l}\text { Cognitive } \\
\text { outcome(s) } \\
\text { measured }\end{array}$ & $\begin{array}{l}\text { Time of } \\
\text { administration } \\
\text { (minutes) }\end{array}$ & Sensitivity (\%) & Specificity & Conclusions & Abbreviations \\
\hline $\begin{array}{l}\text { Cullen et al, } \\
\text { United } \\
\text { Kingdom }\end{array}$ & 36 & $\begin{array}{l}\text {-3MS } \\
\text {-CASI } \\
\text {-MMSE } \\
\text {-SASSI } \\
\text {-STMS } \\
\text {-CAST } \\
\text {-GPCOG } \\
\text {-7MS } \\
\text {-AMT } \\
\text {-Mini-Cog } \\
\text {-SIS } \\
\text {-T\&C } \\
\text {-ACE-R } \\
\text {-DemTect }\end{array}$ & $\begin{array}{l}\text { Gold standard } \\
\text { diagnostic } \\
\text { criteria } \\
\text { (based on } \\
\text { international } \\
\text { diagnostic } \\
\text { guidelines or } \\
\text { clinical judge- } \\
\text { ment fol- } \\
\text { lowing a full } \\
\text { assessment } \\
\text { battery). }\end{array}$ & $\begin{array}{l}\text { Cognitive } \\
\text { impairment } \\
\text { or any type } \\
\text { of dementia }\end{array}$ & $\begin{array}{l}-3 M S=10-15 \\
-\mathrm{CASI}=15-20 \\
-\mathrm{MMSE}=8-13 \\
-\mathrm{SASSI}=10-15 \\
\text {-STMS }=5 \\
-\mathrm{CAST}=15 \\
-\mathrm{GPCOG}=5 \\
-\mathrm{TMS}=7-15 \\
-\mathrm{AMT}=5 \\
-\mathrm{M} \text { Mini-Cog }=3-4 \\
-\mathrm{SIS}=5 \\
-\mathrm{T} \& \mathrm{C}=1 \\
-\mathrm{ACE}-\mathrm{R}=16 \\
-\mathrm{DemTeCt}=8-10\end{array}$ & $\begin{aligned} \text {-3MS }=83-94 \\
\text {-CASI }=91-95 \\
\text {-MMSE }= \\
\quad 69-91 \\
\text {-SASSI }=94 \\
\text {-STMS }=86-95 \\
\text {-CAST }=88-95 \\
\text {-GPCOG }=85 \\
\text {-7MS }=91 \\
\text {-AMT }=73-100 \\
\text {-Mini-Cog }= \\
76-99 \\
\text {-SIS }=81-89 \\
\text {-T\&C = 63-95 } \\
\text {-ACE-R = } \\
84-94 \\
\text {-DemTect } \\
=100 \\
\text { (Alzheimer's } \\
\text { dementia) }\end{aligned}$ & 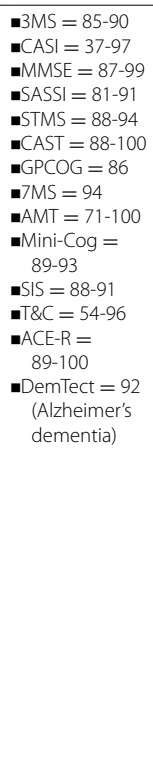 & $\begin{array}{l}\text { These tests were } \\
\text { selected as brief } \\
\text { assessment tools } \\
\text { in the doctor's } \\
\text { office due to } \\
\text { their reported } \\
\text { sensitivity and } \\
\text { specificity values } \\
\text { that were }>85 \% \\
\text { for all dementia } \\
\text { types together } \\
\text { or for more than } \\
\text { one particular } \\
\text { subtype alone, } \\
\text { and/or they } \\
\text { covered at } \\
\text { least three key } \\
\text { domains. } \\
\text { The } 3 \text { MS and CASI } \\
\text { are the only tests } \\
\text { which cover all } \\
\text { six key abilities } \\
\text { (Attention/work- } \\
\text { ing memory, } \\
\text { verbal recall, } \\
\text { expressive } \\
\text { language, verbal } \\
\text { fluency, visual } \\
\text { construction, } \\
\text { reasoning/judge- } \\
\text { ment). }\end{array}$ & $\begin{array}{l}\text { (ACE-R)-Addenbrooke's } \\
\text { Cognitive Examination } \\
\text { Revised, STMS-Short Test } \\
\text { of Mental Status, CCSE- } \\
\text { Cognitive Capacity } \\
\text { Screening Examination, } \\
\text { (R-CAMCOG)-Rotter- } \\
\text { dam Version of the } \\
\text { Cambridge Cognitive } \\
\text { Examination }\end{array}$ \\
\hline $\begin{array}{l}\text { Lischka et al, } \\
\text { Canada }\end{array}$ & 12 & $\begin{array}{l}\text {-MIS } \\
\text {-IST, BVRT } \\
\text {-CAMCI } \\
\text {-ACE } \\
\text {-ADAS-Cog } \\
\text {-CAMCOG } \\
\text {-MoCA } \\
\text {-S-MMSE } \\
\text {-IQCODE } \\
\text {-STMS } \\
\text {-MMSE } \\
\text {-HDS-R } \\
\text {-CCSE }\end{array}$ & $\begin{array}{l}\text { A full clinical } \\
\text { examina- } \\
\text { tion as the } \\
\text { reference } \\
\text { standard. }\end{array}$ & $\begin{array}{l}\text { Dementia, } \mathrm{MCl} \text {, } \\
\text { amnestic } \\
\mathrm{MCl} \text {, mild } \\
\text { demen- } \\
\text { tia, and } \\
\text { questionable } \\
\text { dementia. }\end{array}$ & 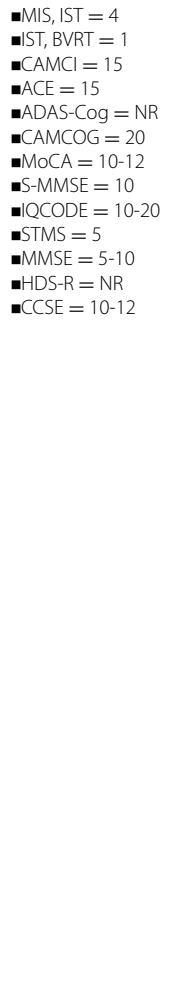 & $\begin{array}{l}\text {-MIS, IST = } 74 \\
\text {-IST, BVRT - } \\
\text { Cutoff level } 1 \\
=90.8 \\
\text {-CAMCI }=83.4 \\
\text {-ACE - Cutoff } \\
<88 / 100= \\
100 \\
\text {-ADAS-Cog } \\
\text { - Cutoff } \\
<75 / 100 \\
=85 \\
\text {-CAMCOG } \\
=76 \text { for } \\
\text { memory } \\
\text { section } \\
\text {-MoCA }=94 \\
\text {-S-MMSE }=14 \\
\text { - IQCODE }=41 \\
\text {-STMS }=\leq 80 \\
\text {-MMSE }=31 \\
\text {-HDS-R }= \\
92 \text { for the } \\
\text { dementia } \\
\text { diabetic } \\
\text { group } \\
\text {-CCSE - Cutoff } \\
26 / 25= \\
88.1\end{array}$ & 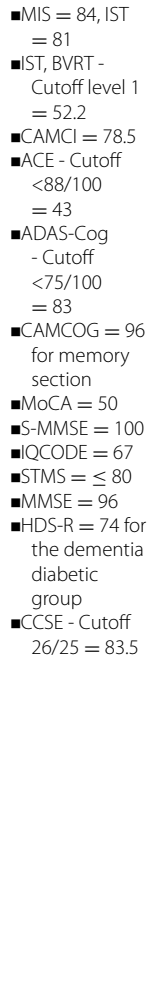 & $\begin{array}{l}\text { Tools with the high- } \\
\text { est specificity } \\
\text { rates: } \\
\text {-MMSE } \\
\text {-S-MMSE } \\
\text { Tests with the } \\
\text { highest sensitivi- } \\
\text { ties: } \\
\text {-HDS-R } \\
\text {-ACE, which } \\
\text { decreased } \\
\text { depending on } \\
\text { cut-off value } \\
\text {-MoCA for the } \\
\text { dementia group } \\
\text { and } 83 \% \text { for the } \\
\text { MCI group } \\
\text {-CAMCI } \\
\text {-CCSE } \\
\text {-The combination } \\
\text { of the MMSE, } \\
\text { IST, and BVRT at } \\
\text { 90.8\% for the first } \\
\text { cut-off level. } \\
\text { The ACE demon- } \\
\text { strated good } \\
\text { diagnostic } \\
\text { accuracy with } \\
\text { AUC=0.98. } \\
\text { Xu et al. (2002) } \\
\text { found that the } \\
\text { CCSE was the } \\
\text { best predictive } \\
\text { screen in MCI } \\
\text { participants for } \\
\text { diagnosing all } \\
\text { dementia due } \\
\text { to its high sen- } \\
\text { sitivity (88.1\%) } \\
\text { and specificity } \\
\text { (83.5\%). }\end{array}$ & $\begin{array}{l}\text { (IST,BVRT)-Isaacs Set Test, } \\
\text { Benton's Visual Reten- } \\
\text { tion Test. CAMCI-Chi- } \\
\text { nese Abbreviated Mild } \\
\text { Cognitive Impairment } \\
\text { Test, (ADAS-Cog)- } \\
\text { Alzheimer Disease } \\
\text { Assessment Scale- } \\
\text { Cognitive Subscale, } \\
\text { (S-MMSE)-Standardized } \\
\text { Mini-Mental State } \\
\text { Examination, (HDS-R)- } \\
\text { Hasegawa Dementia } \\
\text { Scale-Revised, CCSE- } \\
\text { Cognitive Capacity } \\
\text { Screening Examination, } \\
\text { CAMCOG-Cambridge } \\
\text { Cognitive Examination }\end{array}$ \\
\hline
\end{tabular}


Table 1 (continued)

\begin{tabular}{|c|c|c|c|c|c|c|c|c|c|}
\hline $\begin{array}{l}\text { Reference, } \\
\text { Country }\end{array}$ & $\begin{array}{l}\text { Number } \\
\text { of studies } \\
\text { included in } \\
\text { systematic } \\
\text { review }\end{array}$ & Intervention(s) & Comparator & $\begin{array}{l}\text { Cognitive } \\
\text { outcome(s) } \\
\text { measured }\end{array}$ & $\begin{array}{l}\text { Time of } \\
\text { administration } \\
\text { (minutes) }\end{array}$ & Sensitivity (\%) & Specificity & Conclusions & Abbreviations \\
\hline $\begin{array}{l}\text { Boustani et al, } \\
\text { United } \\
\text { States }\end{array}$ & 61 & $\begin{array}{l}\text {-MMSE } \\
\text { - } \mathrm{FAQ} \\
\text {-BIMC } \\
\text {-BOMC } \\
\text {-STMS }\end{array}$ & DSM-IV & Dementia & Not mentioned. & $\begin{array}{l}-\mathrm{MMSE}= \\
71-92 \\
\text {-FAQ }=90 \\
-\mathrm{BIMC}=90 \\
-\mathrm{BOMC}=69 \\
\text {-STMS }=81\end{array}$ & $\begin{array}{l}\text {-MMSE }=56-96 \\
\text { - } F A Q=90 \\
-B I M C=65-90 \\
\text {-BOMC }=90 \\
\text {-STMS }=90\end{array}$ & $\begin{array}{l}\text { The MMSE has } \\
\text { limited Sp when } \\
\text { the cut-point is } \\
\text { set for higher Sn. } \\
\text { Accuracy of the } \\
\text { MMSE changes } \\
\text { based upon the } \\
\text { patients age, } \\
\text { education level } \\
\text { and ethnicity } \\
\text { and therefore } \\
\text { requires adjust- } \\
\text { ment when } \\
\text { used. }\end{array}$ & $\begin{array}{l}\text { BIMC-Blessed Information } \\
\text { Memory Concentration; } \\
\text { BOMC-Blessed Orienta- } \\
\text { tion Memory Concen- } \\
\text { tration; FAQ-Functional } \\
\text { Activities Question- } \\
\text { naire; STMS-Short } \\
\text { Test of Mental Status; } \\
\text { DSM-IV-Diagnostic and } \\
\text { Statistical Manual of } \\
\text { Mental Disorders, fourth } \\
\text { edition }\end{array}$ \\
\hline
\end{tabular}




\section{Results}

The initial search identified 417 unique citations for possible inclusion after duplicates were removed. After searching the reference list of a relevant previous systematic review of systematic reviews [8], three additional citations were collected and screened for eligibility. After screening the 420 citations, 369 were excluded because they did not meet the inclusion criteria. From the 51 full-text articles screened, 30 articles were excluded. Reasons for exclusion include not being a systematic review $(n=20)$, describing a setting other than primary care $(n=1)$, failing to describe the intervention $(n=3)$, or a poor AMSTAR 2 rating $(n=6)$. This resulted in the inclusion of 21 articles (Fig. 1). The included studies were published between June 2003 and July 2019.

\section{Screening tools}

Nine [10-18] out of the 21 included systematic reviews describe screening tools for use in primary care (Table 1). Various screening tools, assessing cognitive impairment or dementia, were compared in terms of cognitive outcomes assessed, time to administer, and sensitivity and specificity. The MMSE was used as a reference standard in the majority of the included studies. The Mini-Cog $(n=5)$ and the MMSE $(n=7)$ were the most widely studied tools among the included reviews. The Mini-Cog takes approximately $3 \mathrm{~min}$ to administer, and sensitivity ranges from 76 to $100 \%$ and specificity from 27 to $93 \%[10,12,14,17]$ depending upon the cutoff value used.

Five systematic reviews examining the MMSE found that it took between 4 and 15 min to administer depending upon the severity of dementia [12-16]. One study found a cut point of 17 had a higher specificity (93\%, $95 \%$ CI: 89-96\%) than a cut point of 24 (46\%, $95 \%$ CI: 40-52\%), while the sensitivity fell from $100 \%$ (95\% CI: $95-100 \%$ ) to $70 \%$ (95\% CI: $59-80 \%$ ) respectively [16].

The Abbreviated Mental Test Score (AMTS) achieved high sensitivity (100\%, $95 \% \mathrm{CI}: 70-100 \%)$ and specificity (82\%, $95 \%$ CI: 72-90\%) [12] compared to a clinical reference standard, and took the shortest amount of time (3.16 to $5 \mathrm{~min})[12,14]$ within primary care. The AMTS was validated for use in general practice [12].

\section{Diagnostic accuracy and physician education}

The diagnosis of dementia by FPs varies but is generally low, as reported in 3 different systematic reviews [11, 16, 19]. In an (urban/rural) study, when following usual practice, only half of cases of mild dementia were diagnosed by the FP [19]. In a separate review, un-diagnosed dementia accounted for $50-66 \%$ of all cases of dementia in three primary care samples studied [11, 20-22]. Another review reported that the recognition of cognitive impairment in usual practice achieved a detection sensitivity of $62.8 \%$ (95\% CI: $38.0-84.4 \%$ ) and specificity of $87.3 \%(n=3 ; 95 \%$ CI: $84.9-89.4 \%)$ [16]. However, medical record notations mentioning dementia were present in only $37.9 \%$ (95\% CI: 26.8-49.6\%) and FPs recorded a definitive dementia diagnosis in the medical record in only $10.9 \%$ (95\% CI: $6.8-15.7 \%$ ) of mild cognitive impairment (MCI) cases [16].

Five of six studies found that FPs had an increased likelihood of suspecting dementia after attending an educational seminar $[23,24]$. One study found that the length of the educational seminar impacted the degree of knowledge about dementia management [24].

\section{Management of dementia}

Decision aids, advanced care planning (ACP), collaboration with a case manager $(\mathrm{CM})$ and practice guidelines are all interventions with variable impact on helping facilitate the management of dementia in primary care [23, 25-29] (Table 2). A CM in particular, such as a nurse specialized in care of older adults, can be an asset to a primary care team with the collective goal of collaborating towards meeting the needs of the patient-caregiver dyad [30]. In the case management intervention group of a randomized controlled trial, neuropsychiatric symptoms of dementia decreased (Mean Effect Size $(\mathrm{MES})=0.88$ ), as well as the numbers of hospital $(\mathrm{MES}=0.66)$ and emergency department admissions $(\mathrm{MES}=0.17)$ [26]. However, it was found that there was a lack of successful implementation of a CM into care teams within primary care because of the absence of CMs within the primary care setting, and $52 \%$ of CMs reported ineffective communication between the CM and FPs [26].

Only one systematic review looked at pharmacological treatments in the context of primary care [11]. There was no clinically important difference observed on neuropsychiatric symptoms between patients with mild to moderate Alzheimer's disease taking cholinesterase inhibitors versus placebo [11].

\section{Supporting caregivers of people with dementia}

FPs reported feeling highly involved in dementia care [31]. However, family caregivers reported that communication with the FPs was unsatisfactory, specifically around awareness of daily care problems (e.g. neuropsychiatric symptoms) [31]. The primary care educational intervention, Resources for Enhancing Alzheimer's Caregiver Health (Department of Veterans Affairs) (REACH $\mathrm{VA})$, involves a trained coach who provides sessions to the caregiver on topics relating to self-care, problem solving, mood management and stress management [32]. 
Table 2 Case management interventions and corresponding comparators and outcomes from the literature included in this systematic review

\begin{tabular}{|c|c|c|c|c|}
\hline Authors, Country & $\begin{array}{l}\text { Number of studies } \\
\text { included in systematic } \\
\text { review }\end{array}$ & Intervention & Comparator & Outcomes \\
\hline Sivananthan et al, Canada & 12 & $\begin{array}{l}7 \text { dementia care processes } \\
\text { recommended by best } \\
\text { practice guidelines: } \\
\text { - Formal memory testing } \\
\text { - Imaging } \\
\text { - Laboratory testing } \\
\text { - Interventions } \\
\text {-Counseling } \\
\text {-Community service } \\
\text { - Specialist referrals }\end{array}$ & $\begin{array}{l}\text { Clinical services provided by } \\
\text { physicians to older adults } \\
\text { diagnosed with dementia. }\end{array}$ & $\begin{array}{l}\text {-8 out of } 12 \text { studies reported } \\
\text { that < } 60 \% \text { of physicians } \\
\text { conducted formal memory } \\
\text { testing, while } 3 \text { studies } \\
\text { reported }<15 \% \text {, and } 1 \text { study } \\
<4 \% \\
\text {-33\% to } 91 \% \text { of family physi- } \\
\text { cian's prescribed medica- } \\
\text { tions for dementia and } \\
\text { consequent behavioral } \\
\text { problems } \\
\text {-33-80\% of physicians } \\
\text { reported the use of CT or } \\
\text { MRI as a diagnostic tool, and } \\
>75 \% \text { used blood work } \\
\text {-2 studies reported that }>80 \% \\
\text { of physicians provided } \\
\text { counseling. }\end{array}$ \\
\hline Khanassov et al, Canada & 23 & $\begin{array}{l}\text { Case Management inter- } \\
\text { ventions comprising all } \\
\text { components identified } \\
\text { by the Case Management } \\
\text { Society of America: } \\
\text {-Case finding and screening } \\
\text {-Assessment } \\
\text {-Care planning } \\
\text { - mplementation and } \\
\text { management } \\
\text {-Monitoring } \\
\text { - Review }\end{array}$ & No comparator & $\begin{array}{l}\text {-Only } 63 \% \text { of case managers } \\
\text { clearly explained their role } \\
\text { to the patient-caregiver } \\
\text { dyads while } 25 \% \text { did not } \\
\text { give any detail during } \\
\text { assessment } \\
\text { - } 52 \% \text { of case managers } \\
\text { indicated that poor com- } \\
\text { munication with health- } \\
\text { care providers negatively } \\
\text { affected their work } \\
\text { - Limiting factors to case } \\
\text { management implemen- } \\
\text { tation were: insufficient } \\
\text { knowledge of diagnostic } \\
\text { tools, absence of training, } \\
\text { and the absence of the case } \\
\text { manager in the primary care } \\
\text { setting. }\end{array}$ \\
\hline
\end{tabular}


Table 2 (continued)

\begin{tabular}{|c|c|c|}
\hline Authors, Country & $\begin{array}{l}\text { Number of studies } \\
\text { included in systematic } \\
\text { review }\end{array}$ & Intervention \\
\hline $\begin{array}{l}\text { Davies et al, United King- } \\
\text { dom }\end{array}$ & 10 & $\begin{array}{l}\text { Decision-making interven- } \\
\text { tions with decision aids in } \\
\text { dementia care (i.e. audio } \\
\text { guided booklet, a printed } \\
\text { decision aids about } \\
\text { dementia and feeding; } \\
\text { a living with dementia } \\
\text { Guiding Options for Living } \\
\text { with Dementia (GOLD) } \\
\text { book; DECIDE interven- } \\
\text { tion: a guided decision } \\
\text { aid participants read and } \\
\text { complete with support } \\
\text { of decision coach to } \\
\text { assist in making decisions } \\
\text { regarding care home } \\
\text { placement, video decision } \\
\text { aid and structured meet- } \\
\text { ing between surrogate } \\
\text { decision maker and } \\
\text { interdisciplinary care plan } \\
\text { team; a video decision aid } \\
\text { and audio description of } \\
\text { advanced dementia) }\end{array}$ \\
\hline
\end{tabular}

Advanced care planning (ACP)

\section{Comparator}

-The majority of studies used a control group - One study used solely listening to a verbal narrative of the disease.

.

o comparator
Outcomes

Place of care:

-DECIDE decreased decisional conflict in caregivers

- GOLD showed less of an increase in burden and greater increase in the knowledge of caregivers Goals of care:

- A video decision aid combined with a structured meeting improved communication between caregivers and professionals and improved the concordance on the goals of care after 9 months

Meta-analysis:

-Two RCTs $(\mathrm{N}=72)$ included.

-Decision aids are effective in decreasing decisional conflict in caregivers (standardized $\mathrm{MD}=-0.50,95 \%$ $\mathrm{Cl}[-0.97,-0.02])$. This suggests increased confidence in decision-making and understanding of the decisions.

-Decisional conflict was measured using the Decision Conflict Scale at 3 months post intervention.

Facilitators for ACP:

- An early start while cognitive decline is mild.

- Inclusion of all stakeholders and a good relationship between the GP, patient, and family carers.

-Discussion of social and medical issues aimed at maintaining a normal life.

-Decision aids that provide information and structure which contribute to decision making.

Barriers for ACP:

- Uncertainty about the timing of ACP.

- How to plan for an uncertain future.

- Lack of knowledge about dementia and patient's lack of knowledge of diagnosis.

-Bad relationships among stakeholders.

- Stress/fear caused by ACP.

-Who should take initiative for ACP.

-Difficulties assessing the dementia patient's decisional capacities.

-Changing preferences. 
Table 2 (continued)

\begin{tabular}{|c|c|c|c|c|}
\hline Authors, Country & $\begin{array}{l}\text { Number of studies } \\
\text { included in systematic } \\
\text { review }\end{array}$ & Intervention & Comparator & Outcomes \\
\hline $\begin{array}{l}\text { Mukadam et al, United } \\
\text { Kingdom }\end{array}$ & 13 & $\begin{array}{l}\text { Interventions intended to } \\
\text { increase the detection of: } \\
\text {-Dementia } \\
\text { - Suspected dementia } \\
\text {-People presenting with } \\
\text { memory complaints }\end{array}$ & $\begin{array}{l}\text { RCT: } \\
\text {-Control groups. } \\
\text { Non-randomized studies } \\
\text { and pre-post study } \\
\text { designs: } \\
\text {-Comparison groups. }\end{array}$ & $\begin{array}{l}\text {-2 of } 3 \text { RCTs of physician } \\
\text { education found group } \\
\text { educational interventions } \\
\text { increased the likelihood } \\
\text { of physicians suspecting } \\
\text { dementia. } \\
\text {-Non-randomized study find- } \\
\text { ings suggest that clinician } \\
\text { education in primary care } \\
\text { interventions can increase } \\
\text { the proportion of patients } \\
\text { in whom physicians suspect } \\
\text { dementia; untargeted com- } \\
\text { munity leaflet campaigns } \\
\text { did not increase dementia } \\
\text { diagnosis rates. } \\
\text {-Pre-post comparison } \\
\text { studies showed no posi- } \\
\text { tive effects for individual } \\
\text { clinician training, group } \\
\text { training with a routine } \\
\text { screening programme or a } \\
\text { targeted leaflet campaign. } \\
\text { An increased number of } \\
\text { memory clinics correlated } \\
\text { with an increased number } \\
\text { of dementia diagnoses. }\end{array}$ \\
\hline
\end{tabular}


Table 2 (continued)

\begin{tabular}{|c|c|c|c|c|}
\hline Authors, Country & $\begin{array}{l}\text { Number of studies } \\
\text { included in systematic } \\
\text { review }\end{array}$ & Intervention & Comparator & Outcomes \\
\hline Khanassov et al, Canada & 43 & $\begin{array}{l}\text { Case management (CM): } \\
\text {-Assessment } \\
\text {-Care planning } \\
\text { - Implementation } \\
\text {-Management } \\
\text { - Regular follow-up }\end{array}$ & $\begin{array}{l}\text { RCT: } \\
\text {-Control group } \\
\text { Qualitative studies: } \\
\text {-No control }\end{array}$ & $\begin{array}{l}\text { RCT evidence: } \\
\text {-4/10 trials showed a } \\
\text { decrease in the frequency } \\
\text { of behavioral symptoms } \\
\text { of dementia in the CM } \\
\text { intervention group (mean } \\
\text { effect size 0.88), while } 2 / 7 \\
\text { reported a decrease in } \\
\text { depression symptoms. } \\
\text { - No effect on cognition } \\
\text { and perceived health was } \\
\text { observed. } \\
\text {-8/11 trials found no effect on } \\
\text { institutionalization. } \\
\text {-Hospital admissions } \\
\text { decreased (MES=0.66) in } \\
\text { 2/5 studies. } \\
\text {-Decreased ER admission } \\
\text { was observed in 1/3 stud- } \\
\text { ies (effect size: } 0.17 \text { ) and } \\
\text { a decrease in length of } \\
\text { hospital stay was shown } \\
\text { in both of the studies that } \\
\text { evaluated this outcome } \\
\text { (MES=1.06). } \\
\text {-For caregivers, 5/10 studies } \\
\text { showed a decrease in } \\
\text { depression (MES=0.68) and } \\
4 / 11 \text { showed a decrease in } \\
\text { burden (MES=0.5). } \\
\text { Barriers to implementation } \\
\text { of CM using outcome } \\
\text { matching: } \\
\text {-Intervention durations being } \\
\text { too short. } \\
\text {-Need for high-intensity CM. } \\
\text { - Scarce communication. } \\
\text {-Case manager and physician } \\
\text { in different locations. } \\
\text { - Lack of healthcare providers } \\
\text { with geriatric training. } \\
\text { Addressing these barriers } \\
\text { correlated with better out- } \\
\text { comes, as studies address- } \\
\text { ing more barriers resulted } \\
\text { in more positive outcomes } \\
\text { (agreement k=0.94; Cl, } \\
0.82-1.1 \text { ). }\end{array}$ \\
\hline
\end{tabular}


Table 2 (continued)

\begin{tabular}{|c|c|c|c|c|}
\hline Authors, Country & $\begin{array}{l}\text { Number of studies } \\
\text { included in systematic } \\
\text { review }\end{array}$ & Intervention & Comparator & Outcomes \\
\hline Perry et al, Netherlands & 6 & $\begin{array}{l}\text { Series of seminars and the } \\
\text { appointment of dementia } \\
\text { care managers. }\end{array}$ & $\begin{array}{l}\text { Control groups in studies: } \\
\text {-Clinical practice guidelines } \\
\text { for dementia received } \\
\text { by mail } \\
\text {-No training } \\
\text { - No seminars } \\
\text {-No training and no } \\
\text { dementia care managers } \\
\text {-Short, partly interactive } \\
\text { seminar on dementia } \\
\text { diagnostics (3 hours). }\end{array}$ & $\begin{array}{l}\text {-Intervention clinics demon- } \\
\text { strated better health-related } \\
\text { quality of life (QoL), overall } \\
\text { quality of health care in } \\
\text { patients, family caregiving } \\
\text { quality, social support and } \\
\text { more family caregivers } \\
\text { reported receiving all the } \\
\text { help they needed. } \\
\text {-The health-related QoL } \\
\text { of the caregiver did not } \\
\text { increase. } \\
\text { - Higher proportions of } \\
\text { patients were newly } \\
\text { diagnosed with dementia } \\
\text { following educational work- } \\
\text { shops and computerized } \\
\text { Decision Support System } \\
\text { (DSS) group compared to } \\
\text { the control group. } \\
\text {-After a 2-h seminar for } \\
\text { physicians there were } \\
\text { higher rates of 'suspected } \\
\text { dementia'and lower rates } \\
\text { of both 'uncertain'and 'non- } \\
\text { suspected'diagnoses when } \\
\text { compared to the control } \\
\text { group. } \\
\text {-Both the mean compliance } \\
\text { per patient to the total set } \\
\text { of } 23 \text { quality indicators, } \\
\text { and the compliance per } \\
\text { indicator for } 21 \text { of } 23 \text { quality } \\
\text { indicators, were better in } \\
\text { intervention clinics than in } \\
\text { control clinics. } \\
\text { - Physicians gained more } \\
\text { knowledge after a 5-h semi- } \\
\text { nar than a 3-h seminar. } \\
\text {-After } 9 \text {-months, more physi- } \\
\text { cians in the intervention } \\
\text { group correctly answered } 2 \\
\text { questions about decision- } \\
\text { making compared to the } \\
\text { control group. Those in the } \\
\text { intervention group more } \\
\text { strongly agreed that 'Older } \\
\text { patients with dementia } \\
\text { are difficult to manage in } \\
\text { primary care'than the PCPs } \\
\text { in the control group. }\end{array}$ \\
\hline
\end{tabular}

REACH VA was successful at increasing carer ability to manage problem behaviours and improved outcomes for caregivers, such as decreased burden, depression and caregiving frustrations [30, 31]. A meta-analysis showed that $58 \%$ (95\% CI: $43-72 \%$ ) of family caregivers were in favor of early dementia diagnosis, $50 \%$ (95\% CI: 35-65\%) needed education on dementia, and $23 \%$ (95\% CI: $17-31 \%)$ needed in-home support [33].

\section{Discussion}

This systematic review of systematic reviews identified evidence to inform processes for diagnosis and management of dementia within primary care. While the diagnostic accuracy of a tool may be high, the time taken to administer the tool and copyright limitation for tool use are also important to consider in the context of a busy primary care office. The MMSE, which is copyrighted, 
may not be the best test for use in general practice. Instead, the AMTS appears to be the most suitable tool for use in a busy primary care office, as it has good diagnostic accuracy, does not appear to be copyright protected and takes less time to administer than the MMSE $[12,14,15]$. The Mini-Cog is also quick to administer, and a Cochrane systematic review evaluating the Mini-Cog across care settings recommended that the Mini-Cog be used initially as a case finding test to identify patients who would benefit from additional cognitive evaluations for dementia [34]. However, the sensitivity of the MiniCog may not be high enough to be considered useful in primary care [17], as too many cases would be missed.

The current literature suggests that the implementation of case management directly into the primary care setting can be of great benefit to the patient-caregiver dyad, as well as to the health care system. The CM can help facilitate the advanced care planning process [29], as well as decrease the frequency of neuropsychiatric symptoms of dementia, symptoms of depression, hospital admissions and length of stay in hospital; caregivers can also benefit by experiencing decreased burden and depression [26]. A Cochrane review evaluating the effectiveness of case management in community settings lends support to dementia case management, finding that carer burden decreased and fewer patients where institutionalized after 6 months [35]. Further, there was a reduction in residential home and hospital use after 6 months of case management implementation [35]. There is however a lack of evidence related to cost effectiveness of case management. Facilitating successful case management and advanced care planning includes early implementation while cognitive decline is mild, involving all stakeholders (caregiver, patient, family and FP), and fostering a good relationship between the $\mathrm{FP}$ and patient-caregiver dyad [29]. The CM should be physically present in the primary care setting, clearly explain their role to all stakeholders, implement high-intensity case management, and communicate frequently to all stakeholders in order to ensure positive outcomes for the patient-caregiver dyad [26, 27].

Combining educational seminars for FPs with dementia case management may be the best management strategy $[23,24]$. Educational interventions focused on dementia diagnosis and management in the context of primary care increased the likelihood of FPs suspecting dementia, while also improving the experience of the family caregiver and the patient $[23,24]$.

There was limited evidence concerning the use of pharmacological interventions for the treatment of dementia within the primary care setting. Unfortunately, many pharmacologic studies do not focus on primary care or FPs, making it difficult to draw conclusions about the approach to take regarding the use of medications in this context. One systematic review found no clinically important differences between groups receiving cholinesterase inhibitors and those receiving a placebo in the development of behavioral and neuropsychiatric symptoms of Alzheimer's disease [11]. Similarly, cholinesterase inhibitor use was found to have uncertain clinical benefit in a recent systematic review that explored the benefits and harms of prescription drugs for the treatment of Alzheimer disease, regardless of care setting [36]. This recent review also found limited benefit for memantine.

\section{Conclusions}

The AMTS is suitable for detecting dementia within primary care given its high sensitivity and short administration time. To improve dementia identification, FPs should participate in educational interventions. Incorporation of CMs into the primary care team can help with dementia management and result in improved outcomes. There is limited evidence supporting the benefit for pharmacological treatments in the context of primary care.

\section{Limitations and Future Research}

A limitation of this systematic review of systematic reviews includes the exclusion of possibly relevant pharmacological reviews, given the fact that we focused on studies conducted in the primary care setting. Future pharmacological studies conducted in the specific context of primary care are needed. Additionally, the results from our review are limited to literature from countries that clearly distinguish primary care from specialist care, given the focus of the search strategy. Lastly, many of the studies included within the identified systematic reviews inappropriately used the MMSE as a reference tool when determining the sensitivity and specificity of various screening tools. Further studies should compare commonly used screening tools within primary care to a recognized gold standard.

\section{Abbreviations \\ FPs: Family physicians; PRISMA: Preferred Reporting Items for Systematic Reviews and Meta-analyses; MMSE: Mini-Mental State Examination; AMTS: Abbreviated Mental Test Score; ACP: Advanced Care Planning; CM: Case Man- ager; MES: Mean Effect Size; REACH VA: Resources for Enhancing Alzheimer's Caregiver Health (Department of Veterans Affairs).}

\section{Supplementary Information}

The online version contains supplementary material available at https://doi. org/10.1186/s12875-021-01461-5.

Additional file 1. 


\section{Acknowledgements}

The authors are grateful to Helen Lee Robertson, MLIS, Health Sciences Library, University of Calgary for assisting in the development of the systematic literature search.

\section{Support}

B. Fernandes was funded as an Undergraduate Summer Research Student by the Division of Geriatric Medicine, University of Calgary and a MITACS studentship. J. Holroyd-Leduc is the University of Calgary Brenda Strafford Foundation Chair in Geriatric Medicine.

\section{Prior Presentation}

None.

\section{Authors' contributions}

All 3 authors derived the study. BF and JHL reviewed all retrieved citations and manuscripts. All 3 authors analysed the findings. BF drafted the manuscript; $\mathrm{JHL}$ and ZG provided critical edits. All 3 authors approved the final version of the manuscript.

\section{Funding}

B. Fernandes was funded as an Undergraduate Summer Research Student by the Division of Geriatric Medicine, University of Calgary and a MITACS studentship. J. Holroyd-Leduc is the University of Calgary Brenda Strafford Foundation Chair in Geriatric Medicine. The funders had no role in the collection, analysis or interpretation of data, or in the preparation of the manuscript.

\section{Availability of data and materials}

All data generated or analysed during this study are included in this published article in Additional file 1: Appendixes 1 and 2.

\section{Declarations}

Ethics approval and consent to participate:

Not applicable.

\section{Consent for publication:}

Not applicable.

\section{Competing interests}

The authors have no conflicts of interest to declare.

\section{Author details}

${ }^{1}$ Faculty of Science, University of Calgary, Calgary, Canada. ${ }^{2}$ Departments of Medicine and Community Health Sciences, Cumming School of Medicine, Foothills Medical Centre, University of Calgary, North Tower (Rm 930), 140329 St NW, Calgary, AB T2N 2T9, Canada.

Received: 9 January 2021 Accepted: 13 May 2021

Published online: 11 August 2021

\section{References}

1. World Health Organization. Dementia WHO. World Health Organization Dementia. Published September 19, 2019. Accessed August 14, 2020. https://www.who.int/news-room/fact-sheets/detail/dementia].

2. Parmar J, Dobbs B, McKay R, et al. Diagnosis and management of dementia in primary care: exploratory study. Can Fam Physician Med Fam Can. 2014;60(5):457-65.

3. Chopra A, Cavalieri TA, Libon DJ. Dementia Screening Tools for the Primary Care Physician. 2007;15(1):9.

4. Ismail Z, Black SE, Camicioli R, et al. Recommendations of the 5th Canadian Consensus Conference on the diagnosis and treatment of dementia. Alzheimers Dement. 2020;16(8):1182-95. https://doi.org/10.1002/alz.12105

5. Pimlott NJG, Persaud M, Drummond N, et al. Family physicians and dementia in Canada: Part 2. Understanding the challenges of dementia care. Can Fam Physician Med Fam Can. 2009:55(5):508-509.e1-7.

6. Moher D, Liberati A, Tetzlaff J, Altman DG, The PRISMA Group. Preferred Reporting Items for Systematic Reviews and Meta-Analyses: The PRISMA
Statement. PLoS Med. 2009;6(7):e1000097. https://doi.org/10.1371/journ al.pmed.1000097

7. Strings attached: CADTH database search filters. Strings attached: CADTH Database Search Filters. Published 2016. Accessed May 11, 2020. https:// www.cadth.ca/resources/finding-evidence/strings-attached-cadthsdatabase-search-filters\#syst

8. Yokomizo JE, Simon SS, de Campos Bottino CM. Cognitive screening for dementia in primary care: a systematic review. Int Psychogeriatr. 2014:26(11):1783-804. https://doi.org/10.1017/S1041610214001082

9. Shea BJ, Reeves BC, Wells G, et al. AMSTAR 2: a critical appraisal tool for systematic reviews that include randomised or non-randomised studies of healthcare interventions, or both. BMJ. Published online September 21, 2017:j4008. https://doi.org/10.1136/bmj.j4008

10. Abd Razak MA, Ahmad NA, Chan YY, et al. Validity of screening tools for dementia and mild cognitive impairment among the elderly in primary health care: a systematic review. Public Health. 2019;169:84-92. https:// doi.org/10.1016/j.puhe.2019.01.001

11. Boustani M, Peterson B, Hanson L, Harris R, Lohr KN. Screening for Dementia in Primary Care: A Summary of the Evidence for the U.S. Preventive Services Task Force. Ann Intern Med. 2003;138(11):927. https:// doi.org/10.7326/0003-4819-138-11-200306030-00015

12. Brodaty $H$, Low L-F, Gibson L, Burns K. What is the best dementia screening instrument for general practitioners to use? Am J Geriatr Psychiatry. 2006;14(5):391-400. https://doi.org/10.1097/01.JGP.0000216181.20416.b2

13. Creavin ST, Wisniewski S, Noel-Storr AH, et al. Mini-Mental State Examination (MMSE) for the detection of dementia in clinically unevaluated people aged 65 and over in community and primary care populations. Cochrane Dementia and Cognitive Improvement Group, ed. Cochrane Database Syst Rev. Published online January 13, 2016. https://doi.org/10. 1002/14651858.CD011145.pub2

14. Cullen B, O'Neill B, Evans JJ, Coen RF, Lawlor BA. A review of screening tests for cognitive impairment. J Neurol Neurosurg Amp Psychiatry. 2007;78(8):790-9. https://doi.org/10.1136/jnnp.2006.095414

15. Lischka AR, Mendelsohn M, Overend T, Forbes D. A Systematic Review of Screening Tools for Predicting the Development of Dementia. Can J Aging Rev Can Vieil. 2012;31(3):295-311. https://doi.org/10.1017/S0714 980812000220

16. Mitchell AJ, Meader N, Pentzek M. Clinical recognition of dementia and cognitive impairment in primary care: a meta-analysis of physician accuracy: Clinical recognition of dementia and mild cognitive impairment. Acta Psychiatr Scand. 2011;124(3):165-83. https://doi.org/10.1111/j.16000447.2011.01730x

17. Seitz DP, Chan CC, Newton HT, et al. Mini-Cog for the diagnosis of Alzheimer's disease dementia and other dementias within a primary care setting. Cochrane Dementia and Cognitive Improvement Group, ed. Cochrane Database Syst Rev. Published online February 22, 2018. https:// doi.org/10.1002/14651858.CD011415.pub2

18. Smith T, Cross J, Poland F, et al. Systematic Review Investigating Multi-disciplinary Team Approaches to Screening and Early Diagnosis of Dementia in Primary Care - What are the Positive and Negative Effects and Who Should Deliver It? Curr Alzheimer Res. 2017;15(1). https://doi.org/10.2174/ 1567205014666170908094931

19. Dungen P, Marwijk HWM, Horst HE, et al. The accuracy of family physicians' dementia diagnoses at different stages of dementia: a systematic review: The accuracy of family physicians' dementia diagnoses. Int J Geriatr Psychiatry. Published online May 2011:n/a-n/a. https://doi.org/10. 1002/gps.2726

20. Eefsting JA, Boersma F, Van den Brink W, Van Tilburg W. Differences in prevalence of dementia based on community survey and general practitioner recognition. Psychol Med. 1996;26(6):1223-30. https://doi.org/10. 1017/s0033291700035947

21. Olafsdóttir M, Skoog I, Marcusson J. Detection of dementia in primary care: the Linköping study. Dement Geriatr Cogn Disord. 2000;11(4):223-9. https://doi.org/10.1159/000017241

22. Valcour VG, Masaki KH, Curb JD, Blanchette PL. The detection of dementia in the primary care setting. Arch Intern Med. 2000;160(19):2964-8. https://doi.org/10.1001/archinte.160.19.2964

23. Mukadam N, Cooper C, Kherani N, Livingston G. A systematic review of interventions to detect dementia or cognitive impairment: Systematic review of interventions to detect dementia. Int J Geriatr Psychiatry. 2015;30(1):32-45. https://doi.org/10.1002/gps.4184 
24. Perry M, Drašković I, Lucassen $P$, Vernooij-Dassen $M$, van Achterberg T, Rikkert MO. Effects of educational interventions on primary dementia care: A systematic review. Int J Geriatr Psychiatry. 2011;26(1):1-11. https://doi. org/10.1002/gps.2479

25. Davies N, Schiowitz B, Rait G, Vickerstaff V, Sampson EL. Decision aids to support decision-making in dementia care: a systematic review. Int Psychogeriatr. 2019;31(10):1403-19. https://doi.org/10.1017/S104161021 9000826

26. Khanassov V, Vedel I, Pluye P. Barriers to Implementation of Case Management for Patients With Dementia: A Systematic Mixed Studies Review. Ann Fam Med. 2014;12(5):456-65. https://doi.org/10.1370/afm.1677

27. Khanassov V, Pluye P, Vedel I. Case management for dementia in primary health care: a systematic mixed studies review based on the diffusion of innovation model. Clin Interv Aging. Published online June 2014:915. https://doi.org/10.2147/CIA.S64723

28. Sivananthan SN, Puyat JH, McGrail KM. Variations in Self-Reported Practice of Physicians Providing Clinical Care to Individuals with Dementia: A Systematic Review. J Am Geriatr Soc. 2013;61(8):1277-85. https://doi.org/ $10.1111 /$ jgs. 12368

29. Tilburgs B, Vernooij-Dassen M, Koopmans R, van Gennip H, Engels Y, Perry M. Barriers and facilitators for GPs in dementia advance care planning: A systematic integrative review. Arendts G, ed. PLOS ONE. 2018;13(6):e0198535. https://doi.org/10.1371/journal.pone.0198535

30. Greenwood N, Pelone F, Hassenkamp A-M. General practice based psychosocial interventions for supporting carers of people with dementia or stroke: a systematic review. BMC Fam Pract. 2016;17(1):3. https://doi.org/ 10.1186/s12875-015-0399-2

31. Schoenmakers B, Buntinx F, Delepeleire J. What is the role of the general practitioner towards the family caregiver of a community-dwelling demented relative?: A systematic literature review. Scand J Prim Health Care. 2009;27(1):31-40. https://doi.org/10.1080/02813430802588907

32. U.S. Department of Veterans Affairs. U.S. Department of Veterans Affairs. Published 2020. https://www.caregiver.va.gov/REACH_VA_Program.asp

33. Khanassov V, Vedel I. Family Physician-Case Manager Collaboration and Needs of Patients With Dementia and Their Caregivers: A Systematic Mixed Studies Review. Ann Fam Med. 2016;14(2):166-77. https://doi.org/ 10.1370/afm.1898

34. Holsinger T, Plassman BL, Stechuchak KM, Burke JR, Coffman CJ, Williams JW. Screening for Cognitive Impairment: Comparing the Performance of Four Instruments in Primary Care. J Am Geriatr Soc. 2012;60(6):1027-36. https://doi.org/10.1111/j.1532-5415.2012.03967.x

35. Reilly S, Miranda-Castillo C, Malouf R, et al. Case management approaches to home support for people with dementia. Cochrane Dementia and Cognitive Improvement Group, ed. Cochrane Database Syst Rev. Published online January 5, 2015. https://doi.org/10.1002/14651858.CD008 345.pub2

36. Fink HA, Linskens EJ, MacDonald R, et al. Benefits and Harms of Prescription Drugs and Supplements for Treatment of Clinical Alzheimer-Type Dementia: A Systematic Review and Meta-analysis. Ann Intern Med. 2020;172(10):656-68. https://doi.org/10.7326/M19-3887

\section{Publisher's Note}

Springer Nature remains neutral with regard to jurisdictional claims in published maps and institutional affiliations.
Ready to submit your research? Choose BMC and benefit from:

- fast, convenient online submission

- thorough peer review by experienced researchers in your field

- rapid publication on acceptance

- support for research data, including large and complex data types

- gold Open Access which fosters wider collaboration and increased citations

- maximum visibility for your research: over $100 \mathrm{M}$ website views per year

At BMC, research is always in progress.

Learn more biomedcentral.com/submissions 